\title{
Evaluation of the Novodiag CarbaR+, a Novel Integrated Sample to Result Platform for the Multiplex Qualitative Detection of Carbapenem and Colistin Resistance Markers
}

\author{
Delphine Girlich, ${ }^{1,2,{ }^{*}}$ Pierre Bogaerts, ${ }^{3,{ }^{*}}$ Warda Bouchahrouf, ${ }^{3}$ Sandrine Bernabeu, ${ }^{1,2,4,5}$ \\ Isabelle Langlois, ${ }^{4}$ Christine Begasse, ${ }^{4}$ Nicolas Arangia, ${ }^{4}$ Laurent Dortet, ${ }^{1,2,4,5}$ \\ Te-Din Huang, ${ }^{3}$ Youri Glupczynski, ${ }^{3}$ and Thierry Naas ${ }^{1,2,4,5}$
}

Objectives: This study evaluated the performance of the Novodiag ${ }^{\circledR}$ CarbaR+ an automated qualitative nucleic acid-based diagnostic assay detecting the $b l a_{\mathrm{VIM}}, b l a_{\mathrm{NDM}}, b l a_{\mathrm{IMP}}, b l a_{\mathrm{OXA}-48}, b l a_{\mathrm{KPC}}, b l a_{\mathrm{OXA}-23}, b l a_{\mathrm{OXA}-58}$, $b l a_{\mathrm{OXA}-24}$, and ISAba1 associated bla $a_{\mathrm{OXA}-51}$ carbapenemase genes and colistin resistance $m c r-1$ gene from clinical isolates or directly from rectal swabs.

Materials and Methods: CarbaR+ was evaluated on 201 clinical isolates and on 100 rectal swabs (80 selected swabs from patients that were evaluated by culture method and/or Xpert Carba-R assay and 20 spiked samples). PCR-sequencing on colonies was considered as the gold standard.

Results: The CarbaR+ detected all the variants of the targeted resistance genes (39 bla $a_{\mathrm{VIM}-}, 30$ bla $_{\mathrm{NDM}}, 20$

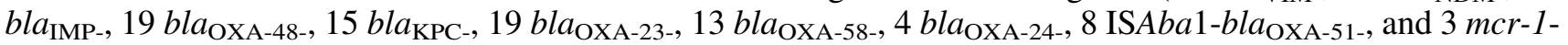
like genes) with sensitivity and specificity of $98.2 \%$ and $99.7 \%$, respectively. On the 80 rectal swabs, 71 CarbaR+ results were fully concordant with the results on selective culture media (66 positive samples with 1 to 3 carbapenemases and 5 negative samples). In eight rectal swabs, CarbaR+ identified additional carbapenemase genes. One false negative result with an Escherichia coli producing-OXA-181 was observed and one CarbaR+ result for OXA-48 was in agreement with Xpert Carba-R assay, without growth on culture media. A concordance of $100 \%$ was observed on spiked samples.

Conclusions: Novodiag CarbaR+ is a random-access fully automated system that achieves excellent performances for the detection of carbapenemase and/or colistin resistance determinants either from cultured clinical isolates or directly from rectal swabs in 80 minutes.

Keywords: Enterobacterales, Acinetobacter, Pseudomonas, carbapenemases

\section{Introduction}

C ARBAPENEMASE-PRODUCING ENTEROBACTERALES (CPE) and nonfermenters (Pseudomonadaceae and Acinetobacter $\mathrm{sp}$.) represent a major public health concern. ${ }^{1,2}$ The most clinically relevant carbapenemases belong to Ambler class A, class B (metallo- $\beta$-lactamases [MBLs]), or class D (Oxacillinases; carbapenem hydrolyzing class $\mathrm{D} \beta$-lactamases [CHDLs]). KPC-type enzymes are the most worldwide disseminated Ambler class A carbapenemases ${ }^{3}$; NDM, VIM, and
IMP-types are the most prevalent Class B carbapenemases (MBLs) ${ }^{4,5}$; and OXA-48-like carbapenemases in Enterobacterales, ${ }^{6}$ OXA-23, OXA-24/-40, OXA-58, OXA-143, the overexpressed chromosomally encoded OXA-51-like enzymes in Acinetobacter sp. ${ }^{7-10}$, and the scarce OXA-198 in Pseudomonas aeruginosa and Enterobacterales ${ }^{11-13}$ represent the main CHDLs.

For serious clinical infections caused by carbapenemresistant Enterobacterales, the use of colistin has become a "last-resort" treatment. ${ }^{14}$ However, the emergence of

\footnotetext{
${ }^{1}$ Team "Resist" UMR1184 "Immunology of Viral, Auto-Immune, Hematological and Bacterial diseases (IMVA-HB)," INSERM, Université Paris-Saclay, CEA, LabEx Lermit, Faculty of Medicine, Le Kremlin-Bicêtre, France.

${ }^{2}$ Evolution and Ecology of Resistance to Antibiotics Unit, Institut Pasteur-APHP-Université Paris-Sud, Paris, France.

${ }^{3}$ Laboratory of Clinical Microbiology, National Reference Center for Monitoring Antimicrobial Resistance in Gram-Negative Bacteria, CHU UCL Namur, Yvoir, Belgium.

${ }_{5}^{4}$ Bacteriology-Hygiene Unit, Assistance Publique-Hôpitaux de Paris, Bicêtre Hospital, Le Kremlin-Bicêtre, France.

${ }^{5}$ Associated French National Reference Center for Antibiotic Resistance: Carbapenemase-Producing Enterobacteriaceae, Le KremlinBicêtre, France.

*These two authors contributed equally to this work.
} 
plasmid-mediated colistin resistance, mostly via the $m c r-1$ gene, also weakens our antimicrobial's armamentarium. ${ }^{15,16}$ So far, 10 plasmid-mediated colistin resistance genes, mcr- 1 to $m c r-10$, have been identified, mostly in Escherichia coli and Salmonella spp. ${ }^{17-20}$ All $\mathrm{mcr}$ genes encode phosphoethanolamine transferases, modifying the lipid A moiety of the lipopolysaccharide (LPS) and leading to a more positively charged LPS structure, and thus to electrostatic repellence of the colistin. ${ }^{21}$

The identification of Enterobacterales expressing both mcr-1- and carbapenemase in human clinical samples in China, ${ }^{22}$ Thailand, ${ }^{23,24}$ Germany, ${ }^{25}$ Portugal, ${ }^{26}$ Italy, ${ }^{27}$ and France $^{28}$ raises concerns about the emergence of nontreatable bacteria and supports the urgent need of detection methods for both kinds of resistance genes.

Optimal detection methods need to be easy, rapid, and accurate to allow rapid implementation of early and adequate infection control measures; it is hence important that the proposed technology could detect carriers directly from rectal swabs avoiding preliminary time-consuming enrichment or culture steps, ideally allowing a random-access (instead of analysis by batch) and including all steps from extraction to results interpretation and protocol edition. Here, we have evaluated a novel diagnostic tool, the Novodiag ${ }^{\circledR}$ CarbaR+ (Mobidiag, Espoo, Finland) targeting genes coding for KPC, VIM, NDM, IMP, OXA-48, OXA-23, -24, -58, and ISAba1-OXA-51 in addition to MCR-1. Time-to-result and price of the Novodiag CarbaR+ were also compared to some other comparable commercially available assays.

\section{Materials and Methods}

\section{Bacterial isolates and clinical rectal swabs}

Bacterial isolates originate from Bicêtre hospital and CHU UCL Namur strain collections. These are representative of the bacterial isolates circulating in France and Belgium over the past 10 years and characterized according to the workflow of each national reference center for antimicrobial resistance. The collection comprised 201 clinical isolates (80 Enterobacterales, 61 Pseudomonas sp., and 60 Acinetobacter sp.) harboring single or multiple carbapenemases or $m c r-1$ genes targeted by the assay, and other resistance markers not targeted by the assay for specificity control (Table S1).

\section{Clinical and spiked rectal swabs}

Over a 6-month period, 80 rectal swabs positive for carbapenemases as detected by culture method and/or Xpert Carba-R assay (Cepheid, Sunnyvale, CA) or originated from high-risk patients or formerly known carriers were collected at the hospital of Bicêtre. The rectal swabs were plated on ChromID Carba Smart (bioMérieux, Marcy-L'Etoile, France) at the clinical microbiology laboratory of the Bicetre hospital. Carbapenemase production was confirmed using NG-Test Carba5 (NG Biotech, Guipry, France) and with the homemade CarbaNP test ${ }^{29}$ and bacterial identification was done using MALDI-TOF (MALDI Biotyper, Bruker). In parallel, the rectal swab samples were further enriched by inoculation of $100 \mu \mathrm{L}$ of the eSwab transport medium (Copan, Brescia, Italy) in Brain Heart Infusion supplemented with $0.5 \mathrm{mg} / \mathrm{L}$ final concentration of Ertapenem and cultured overnight at $37^{\circ} \mathrm{C}$ before plating.

Preliminary tests were carried out to quantify the detection limit of the Novodiag CarbaR+ by inoculating eNat ${ }^{\mathrm{TM}}$ buffer with $10-10^{8} \mathrm{CFU}$ (colony-forming unit) of collection strains. Then, a pool of rectal swabs previously screened as negative on the ChromID CarbaSmart medium and Novodiag CarbaR+ were spiked with $10^{4} \mathrm{CFU}$ of clinical isolates producing rare carbapenemases or rare variants of carbapenemases: KPC (KPC-3, KPC-14, KPC-28, KPC-39), IMP (IMP-4, IMP-8, IMP-14, IMP-58), VIM (VIM-1, VIM-2, VIM-4, VIM-19), one OXA-23 and one OXA-58, three multiple resistant traits mixing different isolates of different species (MCR-1+OXA23; MCR-1+OXA-48; NDM+OXA-23), one OXA-48-like (OXA-517) and one OXA-535 producing Shewanella.

Control in-house PCR were performed as previously reported $^{7,30,31}$ on boiling extracts of isolated colonies grown on the CarbaSmart selective medium (bioMérieux) and PCR products were sequenced with the same specific primers by the Sanger method on an ABI 3130 sequencer (Applied Biosystems).

\section{Ethical approval}

All the rectal samples were left overs of those systematically taken in the hospital to detect multidrug-resistant bacteria to implement the required hygiene control measures and were strictly anonymized. These samples were solely used for what they have been sent for to the bacteriology laboratory.

\section{Detection with Novodiag CarbaR+}

Novodiag CarbaR+ is an integrated sample to result assay with 5 minutes hands-on time and a time-to-result of 80 minutes. The assay cartridge is loaded on the Novodiag instrument that handled every step of the process from sample preparation, DNA amplification, and amplicon detection by array contact fluorescence. Four cartridges can be loaded and run simultaneously. The automated software interpretation of the sample results is based on the validation of internal extraction/inhibition control result. The system is a DNA amplified PCR-based qualitative technology that provides a positive/negative/invalid result for each of the 10 targeted resistance genes families without access to the raw data, amplification curve, or DNA array image. This is hence a "black box" sample to answer technology.

Test samples were prepared by rectal swabs (eSwab, Copan, Italy) or single clinical isolates suspended in eNat tubes (Copan, provided by Mobidiag). For simulated rectal samples, the swab was mixed vigorously and suspended thoroughly in the tube containing eNat solution. The swab was then discarded and the eNat tube was additionally vortexed for about 5 seconds. For bacterial pure culture, one overnight single colony grown on Mueller Hinton agar plate was taken with a sterile loop and directly transferred in eNat. The colony was thoroughly suspended, the loop discarded and the eNat tubed vortexed for about 5 second. Incubation for 30 minutes at room temperature is sufficient for DNA release. Of note, the sample containing eNat buffer-suspended nucleic acid can be transported or stored up to 4 weeks at room temperature. After vortexing, $600 \mu \mathrm{L}$ 
of the eNat suspension were added to the cartridge that was run on the Novodiag system according to the manufacturer's recommendations (Mobidiag, Espoo, Finland).

\section{Statistical analysis}

CarbaR+ results were compared to the reference results obtained by PCR followed by sequencing considered as the gold standard. Performance parameters (sensitivity and specificity) presented here were calculated following resolution of discrepant results taking the total number of targets as denominator. The sensitivity, specificity, positive predictive value (PPV), and negative predictive value (NPV) of Novodiag CarbaR+ assay were calculated with their respective $95 \%$ confidence interval $(95 \% \mathrm{CI})$ using the free online software VassarStats.

\section{Results and Discussion}

The CarbaR+ Novodiag assay has been evaluated on a collection of 201 well characterized clinical isolates of nonfermenters (Pseudomonas sp. $[n=61]$; Acinetobacter $\mathrm{sp}$. $[n=60])$ and Enterobacterales $(n=80)$ expressing various $\beta$ lactamases including 170 resistance genes targeted by the assay (carbapenemases variants $[n=167]$ and $m c r-1[n=3]$ ). The collection also includes 23 carbapenemases and $3 \mathrm{mcr}$ variants that are not targeted by the assay for additional specificity challenge (Table 1 and Supplementary Table S1). In addition, the CarbaR+ Novodiag assay has been evaluated on 80 clinical rectal swabs from patients considered at risk for CPE carriage according to the French Guidelines, for example, patients directly admitted from abroad or previously hospitalized in areas with high prevalence of CPEs (require one screening upon admission), and contact patients of known carriers (Table 2), and on 20 spiked samples.

\section{Performance of CarbaR+ on a collection of well-characterized Enterobacterales, Pseudomonas $s p$. and Acinetobacter sp. isolates}

For the 201 collection isolates, the CarbaR+ kit presents an overall sensitivity, specificity, and PPV and NPV of $98.2 \%$,

Table 1. Strains Collection for the Evaluation of Novodiag CarbaR+

\begin{tabular}{cc}
\hline & Discrepant results \\
Species (No. of isolates) & obtained on the \\
Novodiag system
\end{tabular}
Noncarbapenemase and nontargeted carbapenemase $(n=53)$
Pseudomonas aeruginosa (22), Acinetobacter Overexpressed cephalosporinase, overexpressed baumannii (9), Acinetobacter pittii (3), Enterobacter cloacae (7), Citrobacter freundii (3), other ${ }^{\mathrm{a}}(9)$ ESBLs, OXA-1, OXA-2, OXA-10, OXA- 163, OXA-405, NMCA, Sme, GES, FRI, LMB, TMB, GIM, FIM, SPM, DIM, OXA- 198, OXA-143, OXA-198, OXA-372

Single targeted carbapenemase $(n=117)$ $P$. aeruginosa (30), A baumannii (23), Escherichia coli (25), Klebsiella pneumoniae (11), Pseudomonas putida (5), other (23)

\author{
KPC, VIM, IMP, NDM, OXA-48, \\ ISAba1+OXA-51, OXA-23, OXA-24/40, \\ OXA-58
}

False positives: OXA-163 (1)

OXA-405 (1)

Multiple carbapenemases or colistin targeted resistance $(n=26)$

A baumannii (10), E. coli (5), E. cloacae (3), K. pneumoniae (3), other (5)

Colistin resistance $(n=5)$

E. coli (4), K. pneumoniae (1)

Strains spiked in clinical samples $(n=20)$ Atlantibacter hermannii (1), E. coli (7), K. pneumoniae (3), E. cloacae (3), C. freundii (1) P. aeruginosa (1), A. baumannii (1), Proteus mirabilis (2) Shewanella sp. (1)
KPC+OXA-48, IMP+OXA-58, NDM+ISAbalOXA-51, or OXA-48 or VIM or OXA-23 or KPC, VIM+OXA-48 or OXA-23, OXA23+ISAba1-OXA-51 or OXA-58, MCR$1+\mathrm{NDM}-1$ or OXA-48

MCR-1, MCR-2, MCR-4, MCR-5, PmrB mutation

KPC-3, KPC-14, KPC-28, KPC-39, IMP-4, IMP-8, IMP-14, IMP-58, VIM-1, VIM-2, VIM-4, VIM-19, OXA-23, OXA-58, MCR1+OXA-48, MCR-1+NDM-1, OXA23+NDM-1, OXA-517, OXA-535
False positives:

OXA-23 (1)

OXA-24/40 (1)

False negative: OXA-58 (1)

False negative: OXA-23 (1) ISAbal-OXA-51 (1)

False positive: OXA-58 (1)

Sensitivity $98.2 \%$ (95\% CI: 94.5-99.5)

Specificity $99.7 \%$ (95\% CI: 99.4-99.9)

Positive predictive value 97.1\% (95\% CI: 92.9-98.9)

Negative predictive value $99.8 \%$ (95\% CI: 99.5-99.9)

Very major error: $1(0.6 \%)$

\footnotetext{
a“'Other” are Serratia marcescens, Enterobacter asburiae, Klebsiella oxytoca, Shewanella sp., A. hermannii, P. mirabilis.

bVery major error: lack of detection of OXA-58.

CI, confidence interval; ESBL, extended spectrum $\beta$-lactamase.
} 


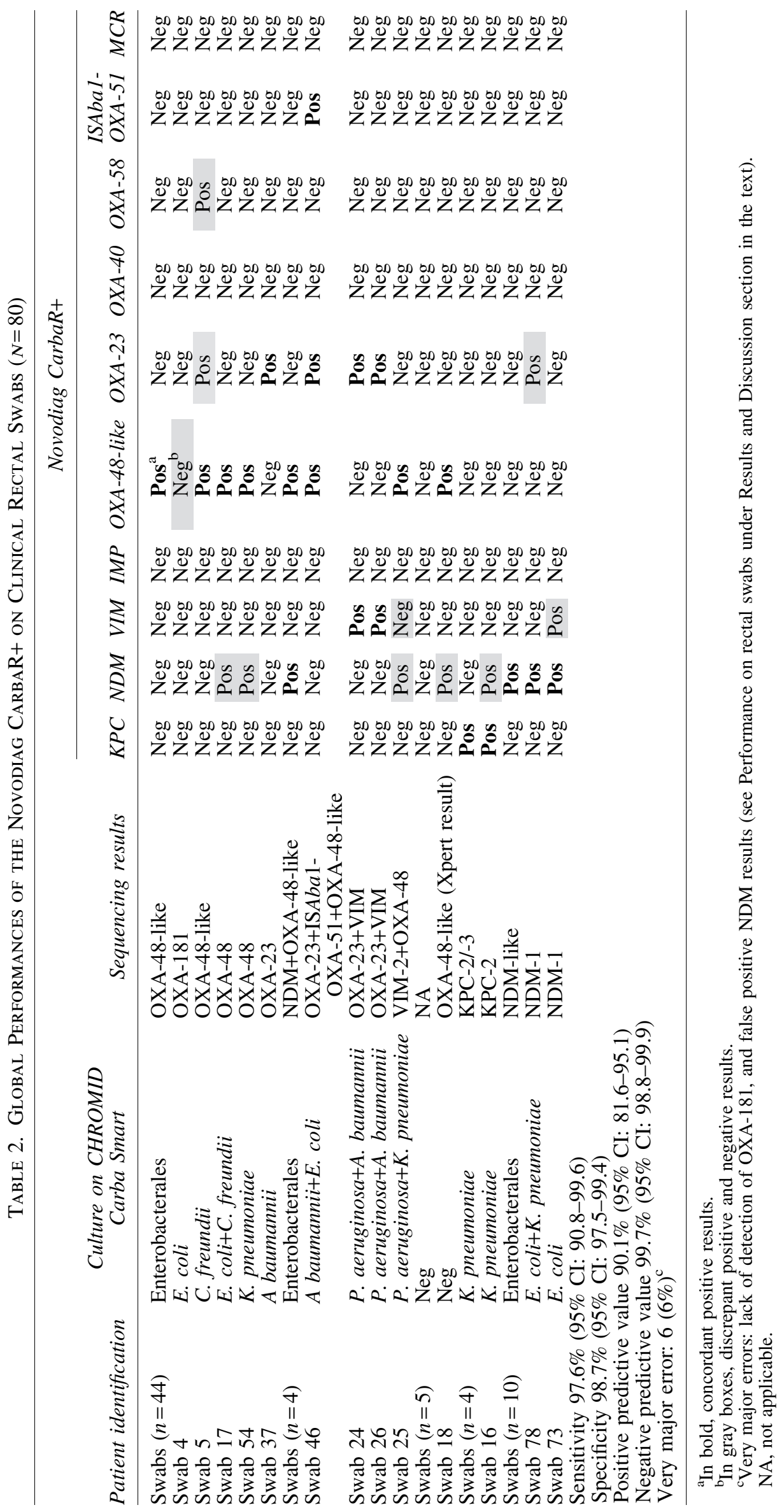


$99.7 \%, 97.1 \%$, and $99.8 \%$, respectively. Five false-positive signals including two OXA-48-like (OXA-163 and OXA-405, which were detected as carbapenemase although considered as extended spectrum $\beta$-lactamase (ESBL) and non-carbapenemase OXA-48 variants), one OXA-23, one OXA-24/40, and one OXA-58 and three false negatives (one OXA-23, one OXA-58, and one ISAbal-OXA-51) were observed.

Whether OXA-163 and OXA-405 are carbapenemases and should be detected as such is still subject of debate. $^{32,33}$ These enzymes are variants of OXA-48 showing much weaker carbapenemase activity when compared with OXA-48, while their activity against ceftazidime is clearly enhanced turning them into OXA-ESBLs than carbapenemases. ${ }^{34,35}$ In specific genetic backgrounds the emergence of carbapenem resistance during carbapenem treatment has been reported for OXA-163-expressing strains. ${ }^{36,37}$ Finally, for CLSI, OXA-163 is still considered as carbapenemase. The situation is getting even more complex with OXA-48like variants (as, e.g., OXA-517, OXA-519) that hydrolyze carbapenems and expanded-spectrum $\beta$-lactams. It is hence important to detect all OXA-48-variants and evaluate their potential impact in clinical management in infection control or antibiotic treatment according to local epidemiology (especially for OXA-163 currently mostly limited in South America). Regarding the three false positive (FP) results in Acinetobacter sp., one bla $a_{\mathrm{OXA}-23}$ gene was detected in a OXA-72-producing (OXA-24/40-like) isolate, one bla $a_{\text {OXA-58 }}$ was detected in a VIM-4+OXA-23 expressing strain that could not be confirmed by end point PCR, ${ }^{38}$ and for one OXA-97-producing Acinetobacter baumannii, bla $a_{\text {OXA-24-like }}$ was detected instead of bla $a_{\text {OXA-58-like. Among false negative }}$ (FN) results observed in Acinetobacter, in one case the $b l a_{\text {OXA-23 }}$ gene was not detected in an OXA-23+OXA-58expressing isolate, in another case, ISAbal-bla $a_{\text {OXA-69 }}$ was not detected in an OXA-23-producing isolate. There could be a lack of detection related to the $b l a_{\text {OXA- } 69}$ allele, as ISAbal-bla $a_{\text {OXA-51 }}$ and ISAbal-bla $a_{\text {OXA-66 }}$ were correctly detected by the assay. The role of ISAbal-bla $a_{\text {OXA-51-like in }}$ carbapenem resistance in A. baumannii is still debated, ${ }^{39,40}$ and as this genetic association is essentially chromosomeencoded, it's potential for dissemination is very limited. Although these results were not correct, they may have little consequences on therapy or on infection control measures.

Among these errors, only one is a major error: the FN OXA-58, which occurred simultaneously with the detection of a FP OXA-24/40. This could not be explained by crossamplification/detection of one OXA with the other because the two bla $_{\text {OXA }}$ genes share less then $50 \%$ sequence identity. ${ }^{10}$ The detection of OXA-24/40 is probably fortuitous and is probably not reproducible. Since no other carbapenemase gene is present in this sample, the consequence of the lack of detection could be the failure to implement hygiene control measures. In terms of groups of microorganisms, a sensitivity and specificity of $100 \%$ was observed for Pseudomonas sp. (P. aeruginosa $[n=53]$, Pseudomonas putida $[n=5]$, Pseudomonas stutzeri $[n=2]$, and Pseudomonas mosselii $[n=1]), 96.7 \%$ and $99.4 \%$ for Acinetobacter (A. baumannii $[n=42]$, Acinetobacter pittii $[n=7]$, and 11 other Acinetobacter sp.), and $100 \%$ and $99.7 \%$ for Enterobacterales, respectively. It is noteworthy that the Car$\mathrm{baR}+$ test was able to detect all different variants of IMP, which is particularly challenging regarding the heterogene- ity of the sequences of this carbapenemase family. In particular, the CarbaR+ test was able to detect $11 b^{\prime} a_{\mathrm{IMP}}$ alleles including IMP-1, IMP-2, IMP-4, IMP-7, IMP-8, IMP-10, IMP-11, IMP-13, IMP-14, IMP-31, and IMP-58 (Supplementary Table S1). CarbaR+ also detected each of the 11 tested variants of OXA-48 with recognized carbapenemase activity including OXA-48, OXA-162, OXA-232, OXA181, OXA-204, OXA-244, OXA-370, OXA-484, OXA-517, OXA-519, and OXA-793. All bla $a_{\mathrm{KPC}}$, bla $_{\mathrm{VIM}}$, and $b l a_{\mathrm{NDM}}$ alleles were also correctly detected (Table 1 and Supplementary Table S1) and as expected only the $m c r-1$ gene was detected by the assay. For isolates harboring multiple carbapenemases $(n=26)$, two signals were missed, but at least one positive signal allowed the bacteria to be correctly categorized as CPO.

\section{Performance on rectal swabs}

Novodiag CarbaR+ is designed for the detection of resistance markers directly on rectal swabs collected for active surveillance of CPE carriers. These results are presented in Table 2. Here, the Novodiag CarbaR+ was evaluated on 80 rectal swabs. The 80 rectal swabs collection included 74 positive and 6 negative samples on CHROMID ${ }^{\circledR}$ Carba Smart agar (bioMérieux). Positive samples contained mostly not only Enterobacterales but also P. aeruginosa and/or Acinetobacter $\mathrm{sp}$. including 48 OXA-48-like, 12 NDM, 5 KPC, 1 OXA-23, and 8 multiple carbapenemases (2 OXA23+VIM, 1 VIM+OXA-48, 2 NDM-5+OXA-181, 2 NDM1+OXA-48, and 1 OXA-23+OXA-48+ISAba1 OXA-51) (Table 2). The latter represented a mix culture containing E. coli OXA-48 and an A. baumannii OXA-23+ISAba1 OXA-51.

Among these 80 clinical screening rectal swabs (Table 2), the overall sensitivity and specificity of the detection of the resistance genes was $97.8 \%$ (95\% CI: 91.1-99.6) and 98.6\% (95\% CI: 97.3-99.3), respectively with 71 CarbaR+ results fully concordant with Carba Smart results and PCR followed by sequencing (66 positive samples for 1-3 carbapenemases and 5 negative samples). For the 9 remaining samples, CarbaR+ identified additional carbapenemase genes in 8 swabs (Table 2), and 1 FN (OXA-181 carrier). The FN OXA-48-like could be explained by a low level of inoculum in the sample, in agreement with the low number of growing colonies on CarbaSmart medium. The resulting NPV was very high: 99.7\% (95\% CI: 98.8-99.9). Interestingly, one positive OXA$48 \mathrm{CarbaR}+$ result corresponded to a patient formerly identified as OXA-48 carrier. In this case, no colony grew on CarbaSmart medium, before and after enrichment broth, nevertheless, Xpert result was also positive. FP results can be explained by the presence of residual DNA in the gut after decolonization of a patient. This is particularly applicable to OXA-23, OXA-51+ISAbal, or VIM in patients who can have been colonized with Acinetobacter sp. (OXA-23 and OXA$51+\mathrm{ISAba1}$ ) or $P$. aeruginosa (VIM). However, the high rate of FP NDM results (5 FP NDM in total) is more troublesome in patients without prior NDM carriage but could also be explained by the presence of low level of NDM-producing $A$. baumannii (Table 2). This high level of FP in the rectal swabs affected the resulting PPV of 90.1\% (95\% CI: 81.6-95.1). The consequence would be that in about $10 \%$ of cases, a positive result would not be linked to the presence of the 
targeted gene. This is particularly annoying in the case of NDM, as these FPs will lead to the lack of use of ceftazidimeavibactam in clinics, although it is now a powerful therapeutic combination against the producers of KPC and OXA-48. ${ }^{1,22}$

\section{Spiked samples}

In addition to clinical samples, CarbaR+ was also challenged with 20 spiked negative swabs. This was done to test for the detection of rarer variants of carbapenemases. Five rectal swabs, confirmed as negative for carbapenemase either by culture or CarbaR+ were spiked with characterized isolates expressing known resistance genes as described in the Material and methods section. These samples comprised four KPC, four IMP, five VIM, OXA-23, OXA-58, OXA517, OXA-535 harboring Shewanella, and three multiple resistant traits mixing different isolates of different species (MCR-1+OXA-23; MCR-1+OXA-48; NDM+OXA-23). All carbapenemases and MCR-1 coding genes were correctly identified by CarbaR+. Only OXA-535 in Shewanella was not detected, which is considered as a true negative. Shewanella sp. carrying various intrinsic chromosome-encoded OXA-48-like variants are, indeed, occasional inhabitants of the fecal flora requiring no specific infection control measures. It is hence favorable if OXA-535 harboring Shewanella was not detected by the CarbaR+ assay, which presents $100 \%$ sensitivity and specificity on spiked samples.

As evaluated on spiked samples, we observed a detection limit of ca. $10^{4} \mathrm{CFU}$ in the rectal swab buffer (eSwab) before introduction in the eNat buffer and in the Novodiag system. This corresponded to a 1/1,000 dilution of a bacterial suspension calibrated to $0.5 \mathrm{McFarland}$.

\section{Comparison with other molecular assays}

Several other assays are commercially available for that purposes. Some require DNA extraction or at least simple sample heating of rectal swabs specimen before molecular analysis (PCR or isothermal Loop-mediated amplification [LAMP]) such as PCR-based Amplidiag ${ }^{\circledR}$ CARBA+MCR assay (Mobidiag, Paris, France), ${ }^{41}$ Amplidiag CARBA+VRE assay (Mobidiag), ${ }^{42}$ the Check-Direct CPE assay (Check-Points, Wageningen, The Netherlands), ${ }^{43}$ or LAMP easyplex ${ }^{\circledR}$ SuperBug panel (Amplex, Gars-Bahnhof, Germany). ${ }^{44,45}$ Others are fully automated, such as CRE ELITe MGB $^{\circledR}$ kits on an InGenius platform (Elitech, Les Ulis, France), ${ }^{46}$ BD MAX ${ }^{\mathrm{TM}} \mathrm{CPO}$ assay (Becton-Dickinson, Le Pont-de-Claix, France), ${ }^{47}$ and Xpert Carba-R assay (Cepheid, Sunnyvale, CA). ${ }^{48}$ All these assays require specific and expensive instrumentation and target mostly the "big 5" carbapenemases: KPC, OXA-48-like, NDM, VIM, and IMP, representing 90-99\% of the carbapenemases produced by Enterobacterales and nonfermenters in Western countries. ${ }^{5}$ We compared the different fully automated and commercially available tests validated on rectal swabs at the level of the cost, hands-on time, random accessibility, and time to results (Table 3). It is important to notice that the prices reported here are catalog prices provided by the French representatives of each company. The performances of the Novodiag CarbaR+ is comparable to other molecular tests, especially Xpert Carba-R assay but targets, in addition to the big 5, OXA-23-like, 24-like, -58-like, ISAbal-bla $a_{\mathrm{OXA}}$ 51-like (more specific of Acinetobacter sp.), and MCR-1. The technology is based on an all-in-one cartridge that is different from other assays, in that it is an end point PCR assay (not a real-time PCR) with a subsequent hybridization step to reveal the amplified PCR products. Thus, results are only available at the end of the 80 minutes, unlike classical realtime PCRs that may get positive already after a few cycles. The specific clinical interest of the targets will remain subject of debate as illustrated by the choice of the panel proposed by all other companies. To that respect, easyplex superbug has chosen to offer different panel comprising a mix of carbapenemases and CTX-M ESBLs or different mixes of class A, B, and D carbapenemases leaving $m c r-1$

Table 3. Comparison of Commercially Available Assays for Carbapenemase Producers Detection in Rectal Swabs

\begin{tabular}{|c|c|c|c|c|c|c|c|c|}
\hline Company & Instrument & $\begin{array}{l}\text { Instrument } \\
\text { price }(€)\end{array}$ & Assay & Targets & $\begin{array}{c}\text { Pricel } \\
\text { Assay }(€)\end{array}$ & $\begin{array}{l}\text { Hands-on } \\
\text { time (min) }\end{array}$ & $\begin{array}{l}\text { Time to } \\
\text { result (min) }\end{array}$ & $\begin{array}{l}\text { Random } \\
\text { access/batch } \\
\text { (No. of } \\
\text { samples } \\
\text { max) }\end{array}$ \\
\hline Cepheid & GenXpert & 56,000 & $\begin{array}{l}\text { Xpert } \\
\text { Carba-R }\end{array}$ & $\begin{array}{l}\text { KPC, IMP, NDM, } \\
\text { VIM, OXA-48 }\end{array}$ & 42.7 & 5 & 45 & RA (4) \\
\hline Amplidiag & Novodiag & 45,000 & CarbaR+ & $\begin{array}{l}\text { KPC, IMP, NDM, } \\
\text { VIM, OXA-48, } \\
\text { OXA-23, -24, } \\
\text {-58, ISAba1- } \\
\text { OXA-51, } \\
\text { MCR-1 }\end{array}$ & 45 & 5 & 80 & RA (4) \\
\hline $\begin{array}{l}\text { Becton } \\
\text { Dickinson }\end{array}$ & $\mathrm{BDMax}^{\mathrm{TM}}$ & 80,000 & $\begin{array}{l}\text { Check-Direct } \\
\text { CPO }\end{array}$ & $\begin{array}{l}\text { KPC, IMP, NDM, } \\
\text { VIM, OXA-48 }\end{array}$ & 10 & 15 & 150 & Batch (24) \\
\hline Elitech & InGenius & 54,900 & $\begin{array}{c}\text { CRE ELITe } \\
\text { MGB }^{\circledR}\end{array}$ & $\begin{array}{l}\text { KPC, IMP, NDM, } \\
\text { VIM, OXA-48 }\end{array}$ & 8 & 15 & 180 & Batch (12) \\
\hline Amplex $^{\mathrm{a}}$ & GenieII & 18,000 & $\begin{array}{c}\text { SuperBug } \\
\text { panel }\end{array}$ & Variable & 10 & 15 & 30 & RA (2) \\
\hline
\end{tabular}

\footnotetext{
${ }^{a}$ Amplex is not a fully automated test but does not necessitate specific facilities and is validated on fecal swabs with 5 minutes heat extraction.
} 
aside in a specific monoplex kit in a test running in max 1530 minutes (max. two samples with random access). Alternatively, lateral flow immunochromatographic assay with a short 2 hours 30 minutes enrichment step achieved 96\% sensitivity for the detection of fecal carriage of OXA-48, KPC, and NDM. This is not significantly longer than Elitech In Genius assay ( 3 hours +20 minutes hands-on time) and do not necessitate any additional instrumentation nor qualified personnel.

\section{Practical considerations of the test and discrepancy resolutions}

The system was very easy to use with a friendly interface. However, several discrepant results were observed when compared to the gold standard (6/201 on colonies, and 16 cartridges on 100 fecal swabs). For three cartridges, more than three positive signals were observed suggesting FP signals that could be resolved by rerunning with a novel cartridge (data not shown because discrepancies have been resolved). According to the manufacturer, this problem could be related to a problem of microfluidic migration, and thus when numerous positive signals appear for a single isolate, careful interpretation of the results is advocated.

Other discrepant results consisted of two samples with one additional unexpected carbapenemase gene (one VIM and one OXA-48). Retesting the sample led to correct identification (data not shown). In one case OXA-519producing Klebsiella pneumoniae, OXA-519 a single point mutation variant of OXA-48, was not detected initially but correctly identified as OXA-48 producers in a second run.

On fecal samples discrepant results occurred at a much higher frequency (16/100 fecal swabs). For these 16 samples, 13 FP results were detected ( 6 for NDM, 3 for OXA48, 2 for OXA-23, and 1 for OXA-58 and VIM), while 7 FN results were observed (1 VIM, 3 OXA-48, 1 OXA-58, and 2 MCR-1). Retesting of these 16 samples confirmed the results in 9 cases (5/6 NDM FP, 1/1 FP VIM, 2/2 FP OXA-23, and 1/1 OXA-58) in addition to $1 / 1 \mathrm{FN}$ VIM and 2/3 FN OXA-48, while for the 7 remaining samples discrepancies were solved, suggesting that some targets could be close to the limit of detection of the assay. As the assay, is based on a black-box system delivering positive or negative signals without access to the raw data, interpretation of the quality of the data is impossible. Thus, an unusual high number of detected targets may need to be retested. Furthermore, as for other molecular assays, $\mathrm{FN}$ results from rectal swabs have been repeatedly identified, strengthening the necessity to continue in parallel the culture of the rectal swabs, as previously observed. ${ }^{49}$

Our study has three shortcomings: the first one being related to the unexplainable rate of FPs, especially with the NDM target; and the second, the limited number of MCR-1 isolates tested. As the main focus was on testing clinical rectal swabs, the prevalence of this target was too low to make truly relevant observations. Furthermore, the relevance of $\mathrm{IS}_{A b a l}$-OXA-51 in imipenem-resistance of $A$. baumannii is still debated and needs to be further addressed.

\section{Conclusion}

The Novodiag CarbaR+ performed with high accuracy either on clinical isolates or on screening rectal swabs. The main advantage of this test is that it offers a large panel of targeted resistance determinants. Indeed, it detects the main carbapenemases encountered in Enterobacterales and Pseudomonas sp. (KPC, NDM, VIM, IMP, and OXA-48like), but it also identifies the most widespread OXAcarbapenemases usually encountered in Acinetobacter sp. (OXA-23-like, OXA-24-like, OXA-58-like, and ISAba1OXA-51), which makes this test unique as compared to most competitors that detect only the five main carbapenemases. Additionally, it targets the most frequent plasmid-mediated colistin-resistance gene $m c r-1$. Secondly, it is a sample in/answer out platform validated on rectal swabs. The Novodiag CarbaR+ presents excellent performances and appears as a real alternative to the Cepheid Xpert Carba-R with comparable price per test (list price: $45 €$ vs. $42.7 €$ for Xpert Carba-R), but a much more interesting price per target (4.5€ vs. $8.54 €$ for Xpert CarbaR).

\section{Disclosure Statement}

All authors disclose any commercial associations that might create a conflict of interest in connection with submitted articles.

\section{Funding Information}

This work was partially funded by the University ParisSaclay, and INSERM, France, and the National Reference Centre of carbapenemases, Belgium. L.D. and T.N. are members of the Laboratory of Excellence in Research on Medication and Innovative Therapeutics (LERMIT) supported by a grant from the French National Research Agency (ANR-10-LABX-33) and by the Joint Programming Initiative on Antimicrobial Resistance (JPIAMR) DesInMBL [ANR-14-JAMR-002].

\section{Supplementary Material}

Supplementary Table S1

\section{References}

1. Elshamy, A.A., and K.M. Aboshanab. 2020. A review on bacterial resistance to carbapenems: epidemiology, detection and treatment options. Future Sci. OA. 6:FSO438.

2. Jean, S.-S., I.M. Gould, W.-S. Lee, P.-R. Hsueh; International Society of Antimicrobial Chemotherapy (ISAC). 2019. New drugs for multidrug-resistant gram-negative organisms: time for Stewardship. Drugs 79:705-714.

3. Munoz-Price, L.S., L. Poirel, R.A. Bonomo, M.J. Schwaber, G.L. Daikos, M. Cormican, G. Cornaglia, J. Garau, M. Gniadkowski, M.K. Hayden, K. Kumarasamy, D.M. Livermore, J.J. Maya, P. Nordmann, J.B. Patel, D.L. Paterson, J. Pitout, M.V. Villegas, H. Wang, N. Woodford, and J.P. Quinn. 2013. Clinical epidemiology of the global expansion of Klebsiella pneumoniae carbapenemases. Lancet Infect. Dis. 13:785-796.

4. Nordmann, P., T. Naas, and L. Poirel. 2011. Global spread of Carbapenemase-producing Enterobacteriaceae. Emerg. Infect. Dis. 17:1791-1798.

5. Nordmann, P., L. Dortet, and L. Poirel. 2012. Carbapenem resistance in Enterobacteriaceae: here is the storm! Trends Mol. Med. 18:263-272.

6. Poirel, L., A. Potron, and P. Nordmann. 2012. OXA-48-like carbapenemases: the phantom menace. J. Antimicrob. Chemother. 67:1597-1606. 
7. Bonnin, R.A., P. Nordmann, and L. Poirel. 2013. Screening and deciphering antibiotic resistance in Acinetobacter baumannii: a state of the art. Expert Rev. Anti Infect. Ther. 11:571-583.

8. Queenan, A.M., and K. Bush. 2007. Carbapenemases: the versatile beta-lactamases. Clin. Microbiol. Rev. 20:440-458.

9. Higgins, P.G., L. Poirel, M. Lehmann, P. Nordmann, and H. Seifert. 2009. OXA-143, a novel carbapenem-hydrolyzing class D $\beta$-lactamase in Acinetobacter baumannii. Antimicrob. Agents Chemother. 53:5035-5038.

10. Poirel, L., S. Marqué, C. Héritier, C. Segonds, G. Chabanon, and P. Nordmann. 2005. OXA-58, a novel class D $\beta$-lactamase involved in resistance to carbapenems in Acinetobacter baumannii. Antimicrob. Agents Chemother. 49:202-208.

11. El Garch, F., P. Bogaerts, C. Bebrone, M. Galleni, and Y. Glupczynski. 2011. OXA-198, an acquired carbapenemhydrolyzing class D beta-lactamase from Pseudomonas aeruginosa. Antimicrob. Agents Chemother. 55:4828-4833.

12. Bonnin, R.A., P. Bogaerts, D. Girlich, T.-D. Huang, L. Dortet, Y. Glupczynski, and T. Naas. 2018. Molecular characterization of OXA-198 carbapenemase-producing Pseudomonas aeruginosa clinical isolates. Antimicrob. Agents Chemother. 62:e2496-17.

13. Bonnin, R.A., A.B. Jousset, L. Gauthier, C. Emeraud, D. Girlich, A. Sauvadet, G. Cotellon, T. Jové, L. Dortet, and T. Naas. 2020. First occurrence of the OXA-198 carbapenemase in Enterobacterales. Antimicrob. Agents Chemother. 64:e01471-19.

14. Falagas, M.E., P. Lourida, P. Poulikakos, P.I. Rafailidis, and G.S. Tansarli. 2014. Antibiotic treatment of infections due to carbapenem-resistant Enterobacteriaceae: systematic evaluation of the available evidence. Antimicrob. Agents Chemother. 58:654-663.

15. Liu, Y.-Y., Y. Wang, T.R. Walsh, L.-X. Yi, R. Zhang, J. Spencer, Y. Doi, G. Tian, B. Dong, X. Huang, L.F. Yu, D. Gu, H. Ren, X. Chen, L. Lv, D. He, H. Zhou, Z. Liang, J.H. Liu, and J. Shen. 2016. Emergence of plasmid-mediated colistin resistance mechanism MCR-1 in animals and human beings in China: a microbiological and molecular biological study. Lancet Infect. Dis. 16:161-168.

16. Xavier, B.B., C. Lammens, R. Ruhal, S. Kumar-Singh, P. Butaye, H. Goossens, and S. Malhotra-Kumar. 2016. Identification of a novel plasmid-mediated colistinresistance gene, $m c r$-2, in Escherichia coli, Belgium, June 2016. Euro Surveill. 21:30280.

17. Yin, W., H. Li, Y. Shen, Z. Liu, S. Wang, Z. Shen, R. Zhang, T.R. Walsh, J. Shen, and Y. Wang. 2017. Novel plasmid-mediated colistin resistance gene $\mathrm{mcr}-3$ in Escherichia coli. mBio. 8:e0543-17.

18. Carattoli, A., L. Villa, C. Feudi, L. Curcio, S. Orsini, A. Luppi, G. Pezzotti, and C.F. Magistrali. 2017. Novel plasmid-mediated colistin resistance $m c r-4 g$ ene in Salmonella and Escherichia coli, Italy 2013, Spain and Belgium, 2015 to 2016. Euro Surveill. 22:30589.

19. Borowiak, M., J. Fischer, J.A. Hammerl, R.S. Hendriksen, I. Szabo, and B. Malorny. 2017. Identification of a novel transposon-associated phosphoethanolamine transferase gene, mcr-5, conferring colistin resistance in d-tartrate fermenting Salmonella enterica subsp. enterica serovar Paratyphi B. J. Antimicrob. Chemother. 72:3317-3324.

20. Wang, C., Y. Feng, L. Liu, L. Wei, M. Kang, and Z. Zong. 2020. Identification of novel mobile colistin resistance gene mcr-10. Emerg. Microbes Infect. 9:508-516.
21. Poirel, L., A. Jayol, and P. Nordmann. 2017. Polymyxins: antibacterial activity, susceptibility testing, and resistance mechanisms encoded by plasmids or chromosomes. Clin. Microbiol. Rev. 30:557-596.

22. Du, H., L. Chen, Y.-W. Tang, and B.N. Kreiswirth. 2016. Emergence of the mcr-1 colistin resistance gene in carbapenem-resistant Enterobacteriaceae. Lancet Infect. Dis. 16:287-288.

23. Srijan, A., K.R. Margulieux, S. Ruekit, E. Snesrud, R. Maybank, O. Serichantalergs, R. Kormanee, P. Sukhchat, J. Sriyabhaya, M. Hinkle, J.M. Crawford, P. McGann, and B.E. Swierczewski. 2018. Genomic characterization of nonclonal mcr-1-positive multidrug-resistant Klebsiella pneumoniae from clinical samples in Thailand. Microb. Drug Resist. 24:403-410.

24. Paveenkittiporn, W., A. Kerdsin, S. Chokngam, C. Bunthi, S. Sangkitporn, and C.J. Gregory. 2017. Emergence of plasmid-mediated colistin resistance and New Delhi metallo- $\beta$-lactamase genes in extensively drug-resistant Escherichia coli isolated from a patient in Thailand. Diagn. Microbiol. Infect. Dis. 87:157-159.

25. Falgenhauer, L., S.-E. Waezsada, Y. Yao, C. Imirzalioglu, A. Käsbohrer, U. Roesler, G.B. Michael, S. Schwarz, G. Werner, L. Kreienbrock, T. Chakraborty; RESET consortium. 2016. Colistin resistance gene $\mathrm{mcr}-\mathrm{l}$ in extendedspectrum $\beta$-lactamase-producing and carbapenemaseproducing Gram-negative bacteria in Germany. Lancet Infect. Dis. 16:282-283.

26. Mendes, A.C., Â. Novais, J. Campos, C. Rodrigues, C. Santos, P. Antunes, H. Ramos, and L. Peixe. 2018. mcr-1 in carbapenemase-producing Klebsiella pneumoniae with Hospitalized Patients, Portugal, 2016-2017. Emerg. Infect. Dis. 24:762-766.

27. Di Pilato, V., F. Arena, C. Tascini, A. Cannatelli, L. Henrici De Angelis, S. Fortunato, T. Giani, F. Menichetti, and G.M. Rossolini. 2016. $m c r-1.2$, a new $m c r$ variant carried on a transferable plasmid from a colistin-resistant KPC carbapenemase-producing Klebsiella pneumoniae strain of sequence type 512. Antimicrob. Agents Chemother. 60:5612-5615.

28. Beyrouthy, R., F. Robin, A. Lessene, I. Lacombat, L. Dortet, T. Naas, V. Ponties, and R. Bonnet. 2017. MCR-1 and OXA-48 in vivo acquisition in KPC-producing Escherichia coli after colistin treatment. Antimicrob. Agents Chemother. 61:e02540-16.

29. Dortet, L., L. Poirel, and P. Nordmann. 2012. Rapid identification of carbapenemase types in Enterobacteriaceae and Pseudomonas spp. by using a biochemical test. Antimicrob. Agents Chemother. 56:6437-6440.

30. Naas, T., G. Cuzon, P. Bogaerts, Y. Glupczynski, and P. Nordmann. 2011. Evaluation of a DNA microarray (Check-MDR CT102) for rapid detection of TEM, SHV, and CTX-M extended-spectrum-lactamases and of KPC, OXA-48, VIM, IMP, and NDM-1 carbapenemases. J. Clin. Microbiol. 49:1608-1613.

31. Gauthier, L., R.A. Bonnin, L. Dortet, and T. Naas. 2017. Retrospective and prospective evaluation of the Carbapenem inactivation method for the detection of Carbapenemase-producing Enterobacteriaceae. PLoS One 12:e0170769.

32. Dortet, L., and T. Naas. 2017. Noncarbapenemase OXA-48 variants (OXA-163 and OXA-405) falsely detected as Carbapenemases by the $\beta$ Carba test. J. Clin. Microbiol. 55: 654-655. 
33. Arlet, G., D. Decré, M. Lavollay, and I. Podglajen. 2017. Reply to "Noncarbapenemase OXA-48 Variants (OXA163 and OXA-405) Falsely Detected as Carbapenemases by the $\beta$ Carba Test." J. Clin. Microbiol. 55:656-657.

34. Dortet, L., S. Oueslati, K. Jeannot, D. Tandé, T. Naas, and P. Nordmann. 2015. Genetic and biochemical characterization of OXA-405, an OXA-48-type extended-spectrum $\beta$-lactamase without significant carbapenemase activity. Antimicrob. Agents Chemother. 59:3823-3828.

35. Oueslati, S., P. Retailleau, L. Marchini, L. Dortet, R.A. Bonnin, B.I. Iorga, and T. Naas. 2019. Biochemical and structural characterization of OXA-405, an OXA-48 variant with extended-spectrum $\beta$-lactamase activity. Microorganisms. 8:E24.

36. Abdelaziz, M.O., C. Bonura, A. Aleo, R.A. El-Domany, T. Fasciana, and C. Mammina. 2012. OXA-163-producing Klebsiella pneumoniae in Cairo, Egypt, in 2009 and 2010. J. Clin. Microbiol. 50:2489-2491.

37. Pasteran, F., L. Denorme, I. Ote, S. Gomez, D. De Belder, Y. Glupczynski, P. Bogaerts, B. Ghiglione, P. Power, P. Mertens, and A. Corso. 2016. Rapid identification of OXA-48 and OXA-163 subfamilies in Carbapenem-resistant Gram-negative Bacilli with a novel immunochromatographic lateral flow assay. J. Clin. Microbiol. 54:2832-2836.

38. Bonnin, R.A., M. Levy, G. Cuzon, L. Dortet, and T. Naas. 2019. Carbapenemase-producing Acinetobacter spp. from environmental sources in a hospital in French Polynesia. J. Glob. Antimicrob. Resist. 16:81-82.

39. Pagano, M., A.F. Martins, A.B.M.P. Machado, J. Barin, and A.L. Barth. 2013. Carbapenem-susceptible Acinetobacter baumannii carrying the IS Abal upstream bla $a_{\text {OXA-51-like }}$ gene in Porto Alegre, southern Brazil. Epidemiol. Infect. 141:330-333.

40. Sen, B., and S.G. Joshi. 2016. Studies on Acinetobacter baumannii involving multiple mechanisms of carbapenem resistance. J. Appl. Microbiol. 120:619-629.

41. Girlich, D., S. Bernabeu, V. Grosperrin, I. Langlois, C. Begasse, N. Arangia, E. Creton, G. Cotellon, A. Sauvadet, L. Dortet, and T. Naasl. 2018. Evaluation of the Amplidiag CarbaR+MCR kit for accurate detection of Carbapenemase-producing and Colistin-resistant bacteria. J. Clin. Microbiol. 57:e01800-18.

42. Oueslati, S., D. Girlich, L. Dortet, and T. Naas. 2018. Evaluation of the Amplidiag CarbaR+VRE kit for accurate detection of Carbapenemase-producing bacteria. J. Clin. Microbiol. 56:e01092-17.

43. Lau, A.F., G.A. Fahle, M.A. Kemp, A.N. Jassem, J.P. Dekker, and K.M. Frank. 2015. Clinical performance of Check-Direct CPE, a multiplex PCR for direct detection of bla KPC, bla NDM and/or bla vIM, and bla oxA-48 from perirectal swabs. J. Clin. Microbiol. 53:3729-3737.

44. Sękowska, A., T. Bogiel, and E. Gospodarek-Komkowska. 2020. Evaluation of eazyplex ${ }^{\circledR}$ SuperBug CRE test for beta-lactamase genes detection in Klebsiella spp. and $P$. aeruginosa strains. Curr. Microbiol. 77:99-103.

45. Zalas-Więcek, P., E. Gospodarek-Komkowska, and A. Smalczewska. 2020. Rapid detection of genes encoding extended-spectrum beta-lactamase and Carbapenemase in clinical Escherichia coli isolates with eazyplex SuperBug CRE system. Microb. Drug Resist. 26:12451249.

46. Girlich, D., S. Bernabeu, N. Fortineau, L. Dortet, and T. Naas. 2018. Evaluation of the CRE and ESBL ELITe $\mathrm{MGB}^{\circledR}$ kits for the accurate detection of carbapenemase- or CTX-M-producing bacteria. Diagn. Microbiol. Infect. Dis. 92:1-7.

47. Girlich, D., S. Oueslati, S. Bernabeu, I. Langlois, C. Begasse, N. Arangia, E. Creton, G. Cotellon, A. Sauvadet, L. Dortet, N. Fortineau, and T. Naas. 2020. Evaluation of the BD MAX Check-Points CPO assay for the detection of Carbapenemase producers directly from rectal swabs. J. Mol. Diagn. 22:294-300.

48. Cury, A.P., J.N. Almeida Junior, S.F. Costa, M.C. Salomão, Í. Boszczowski, A.J.S. Duarte, and F. Rossi. 2020. Diagnostic performance of the Xpert Carba- $\mathrm{R}^{\mathrm{TM}}$ assay directly from rectal swabs for active surveillance of carbapenemaseproducing organisms in the largest Brazilian University Hospital. J. Microbiol. Methods. 171:105884.

49. Girlich, D., S. Ouzani, I. Langlois, C. Begasse, N. Arangia, N. Fortineau, T. Naas, and L. Dortet. 2020. Successful use of culture and enrichment for the detection of OXA-181producing Escherichia coli from rectal swab samples falsely categorized as negative by Xpert $^{\circledR}$ Carba-R. Diagn. Microbiol. Infect. Dis. 96:114909.

Address correspondence to: Delphine Girlich, PhD Team “Resist”' UMR1184 "Immunology of Viral, Auto-Immune, Hematological and Bacterial diseases (IMVA-HB)',

INSERM, Université Paris-Saclay, CEA, LabEx Lermit, Faculty of Medicine

Hôpital de Bicêtre 78 rue du Général Leclerc Le Kremlin-Bicêtre 94270

France

E-mail: dgirlich@yahoo.fr 